\title{
Answer to a Quiz on page 24: Echocardiography
}

Correct Answer is C

Irregular heart rhythm is clearly seen in the ECG recording (white asterisks). The patient had atrial fibrillation (Fig. 1a)
A prominent systolic flow waves toward the transducer were noted (arrows) (Fig. 1b). Diastolic flow waves away from the transducer were also recorded (red asterisks) (Fig. 1c).

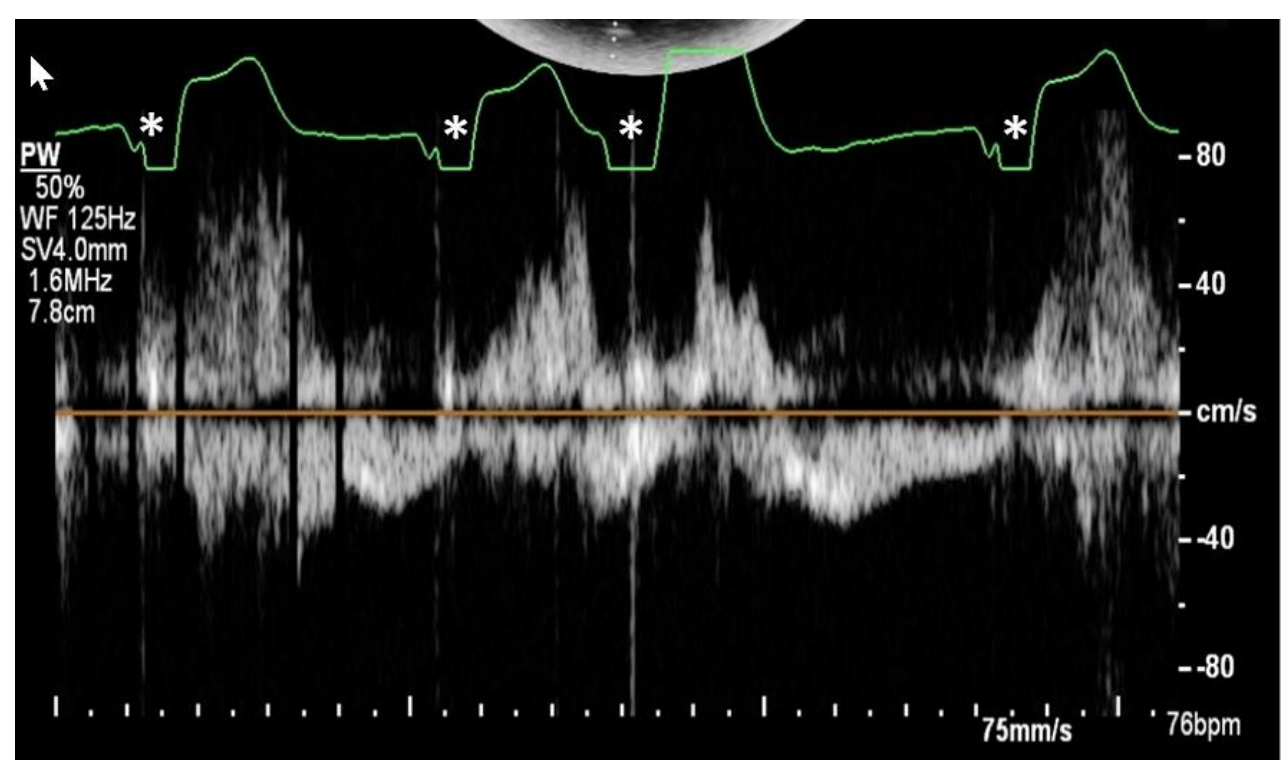

A)

Figure 1. A. Irregular heart rhythm: atrial fibrillation

Address for Correspondence: Oben Baysan, Guven Hospital, Ankara, Turkey,

Email: obbaysan@gmail.com

Received: 02.02.2019 Accepted: 03.02.2019

Copyright@2019 Heart, Vessels and Transplantation Doi: 10.24969/hvt.2019.104 
Heart, Vessels and Transplantation 2019; 3: Doi: 10.24969/hvt.2019.104

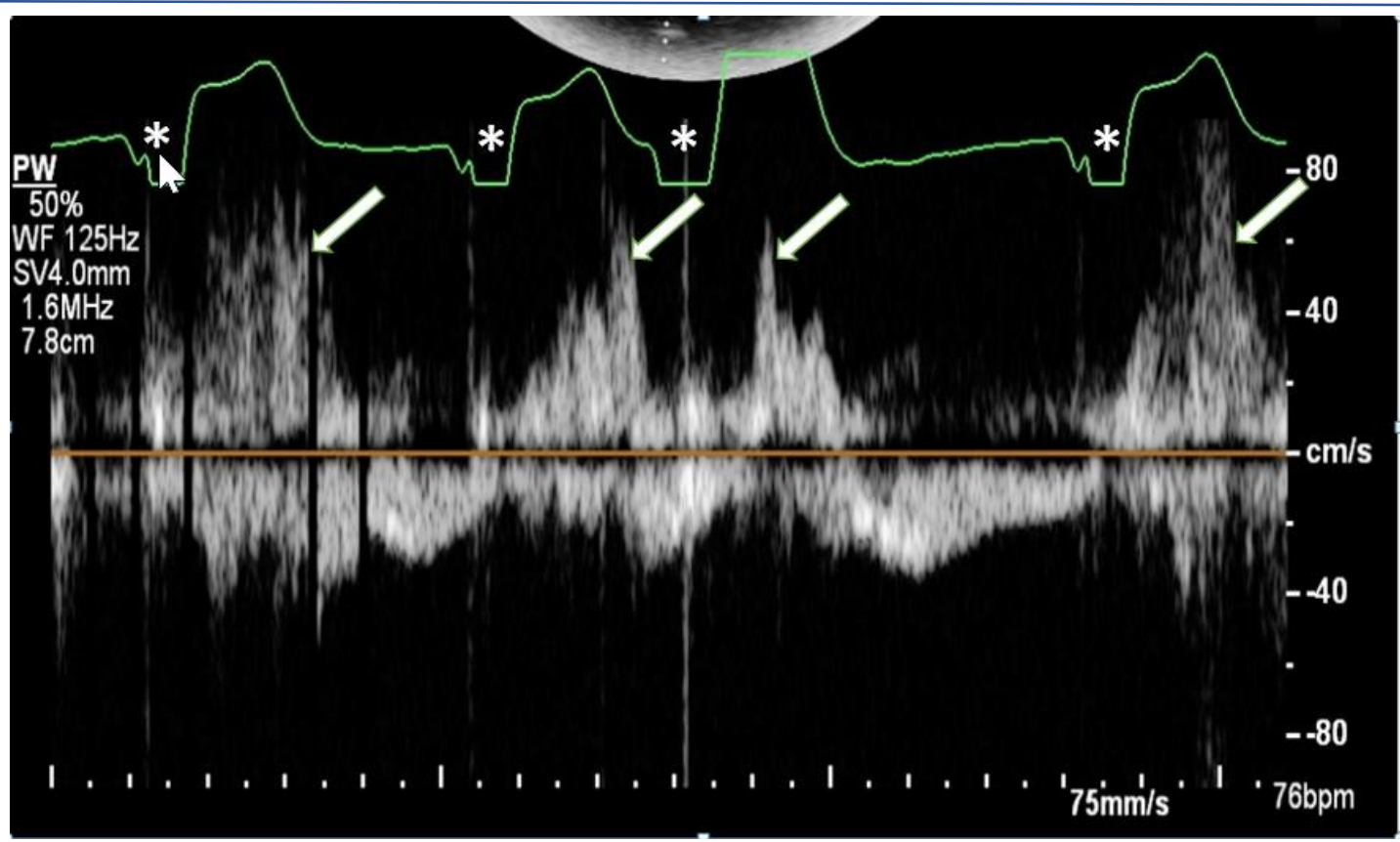

B)

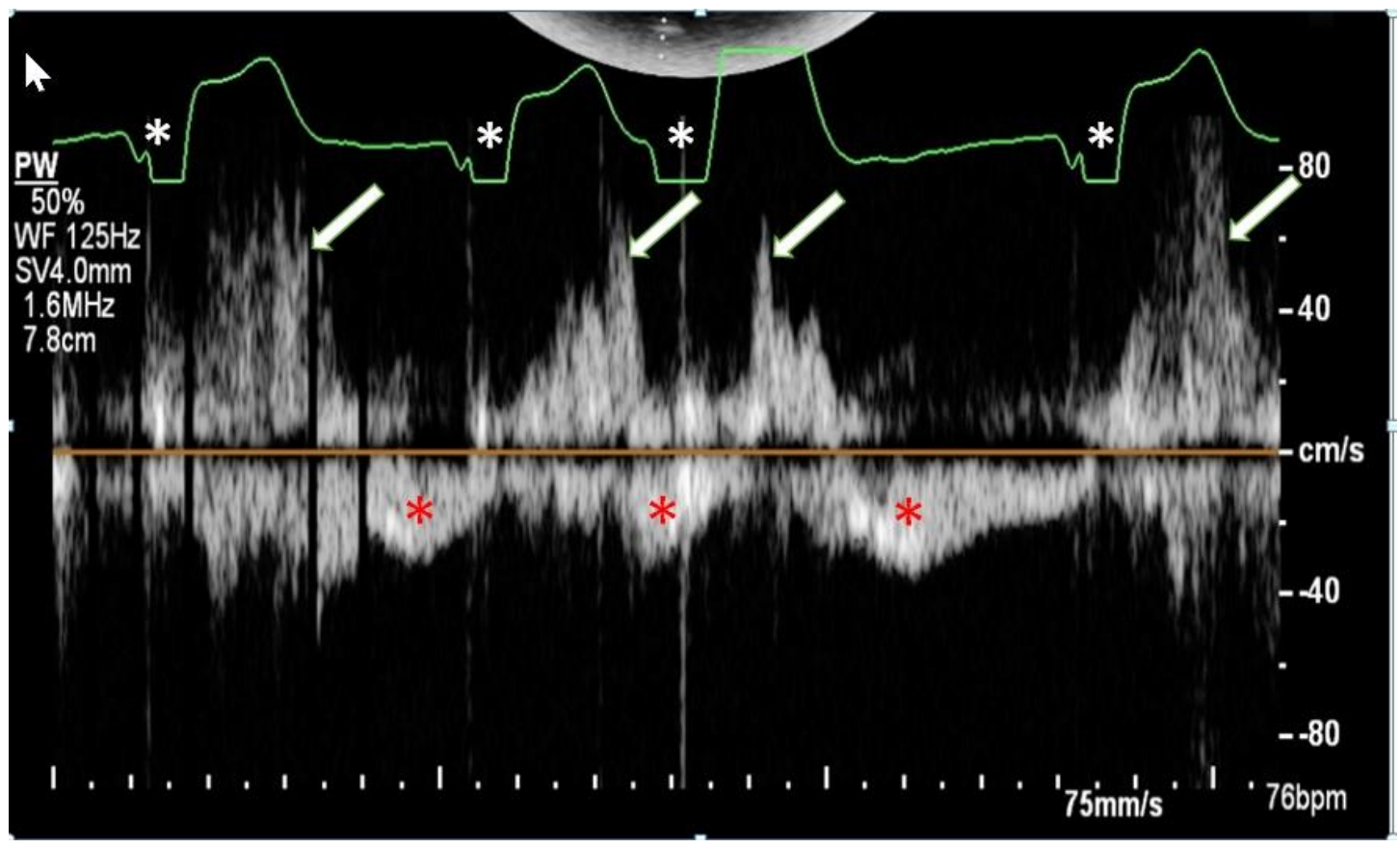

C)

Figure 1. B. Systolic turbulent flow waves; C. Diastolic flow waves 
Hepatic vein flow has four distinct waves: forward flow waves towards right atria: systolic (S) and diastolic (D); backward flows towards the transducer: late systolic $\mathrm{V}$ and late diastolic A waves (Fig. 2).

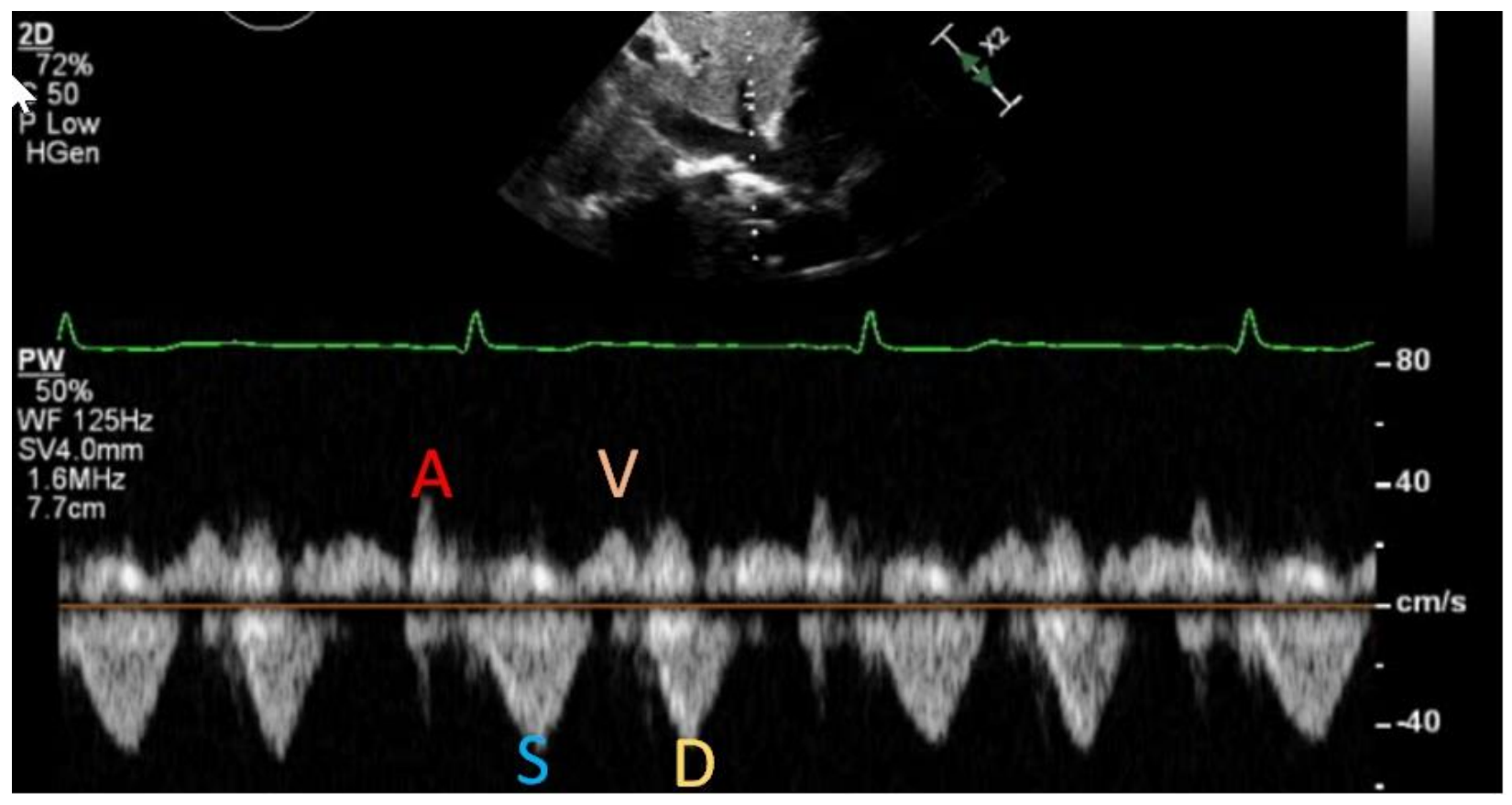

Figure 2. Normal hepatic vein flow

In atrial fibrillation the hepatic vein retrograde $A$ wave is absent as expected. Forward $\mathrm{S}$ wave is blunted and D wave predominates (1). High right ventricular filing pressures lead to even more suppression or reversing of $S$ wave (1). Severe tricuspid regurgitation is a well-known factor causing systolic hepatic vein systolic backward flow (2).
In our case, the patient had all of the above factors affecting hepatic vein flow: atrial fibrillation, high right ventricular pressures and severe tricuspid regurgitation (Movie 1).

Contrary to systolic flow reversal in our case diastolic tricuspid regurgitation could be expected in complete heart block (Fig. 3) (3). 


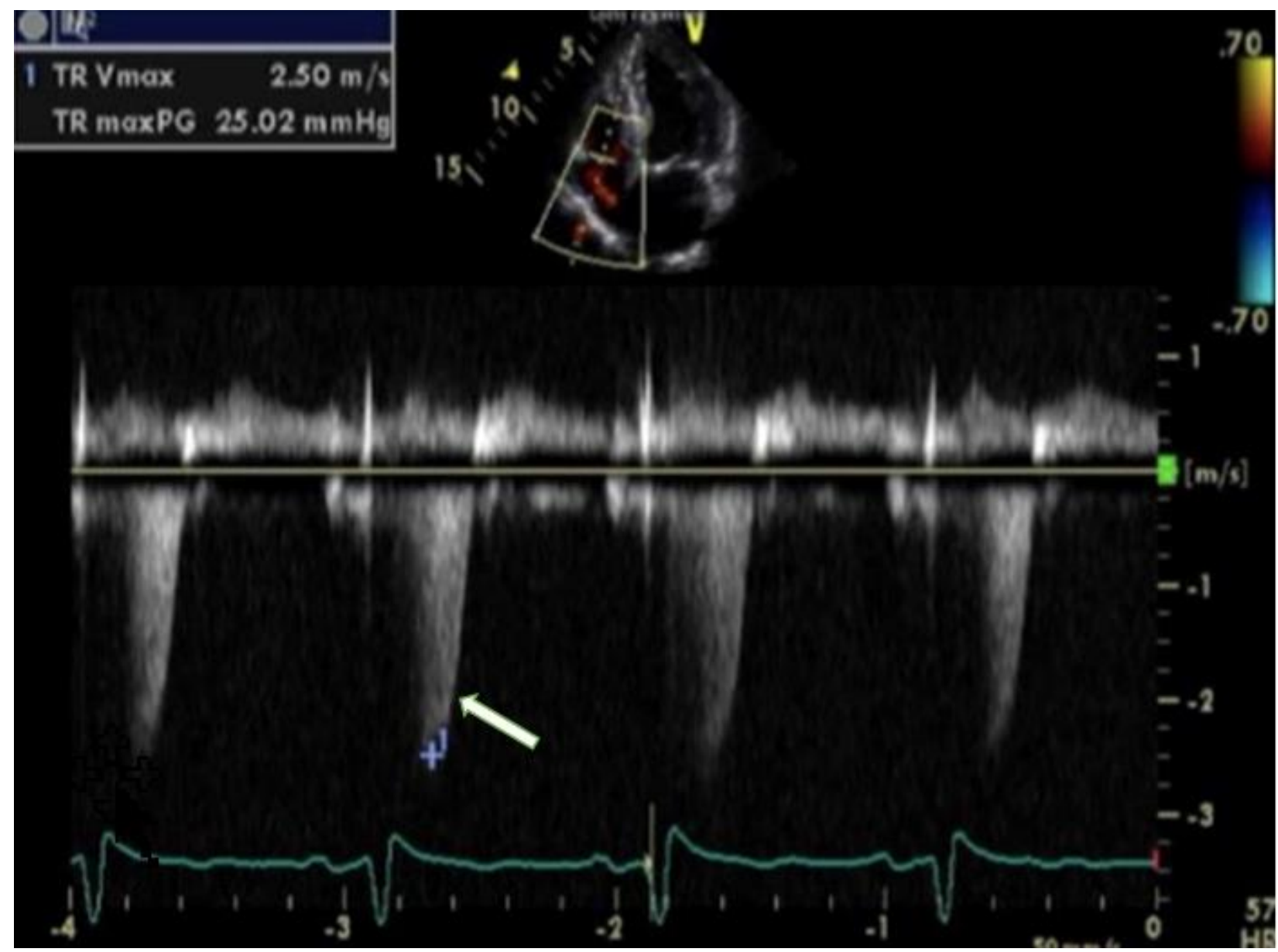

Figure 3. Diastolic tricuspid regurgitation in complete heart block (3)

Normal pulmonary vein flow contains systolic component followed by a diastolic component (D). During atrial contraction, there is slight flow reversal (Ar) (Fig. 4). In our case, diastolic flow waves were clearly seen (red asterisks). Therefore, there is no chance for those diastolic waves to be taken as $\mathrm{Ar}$ waves in the presence of atrial fibrillation. 


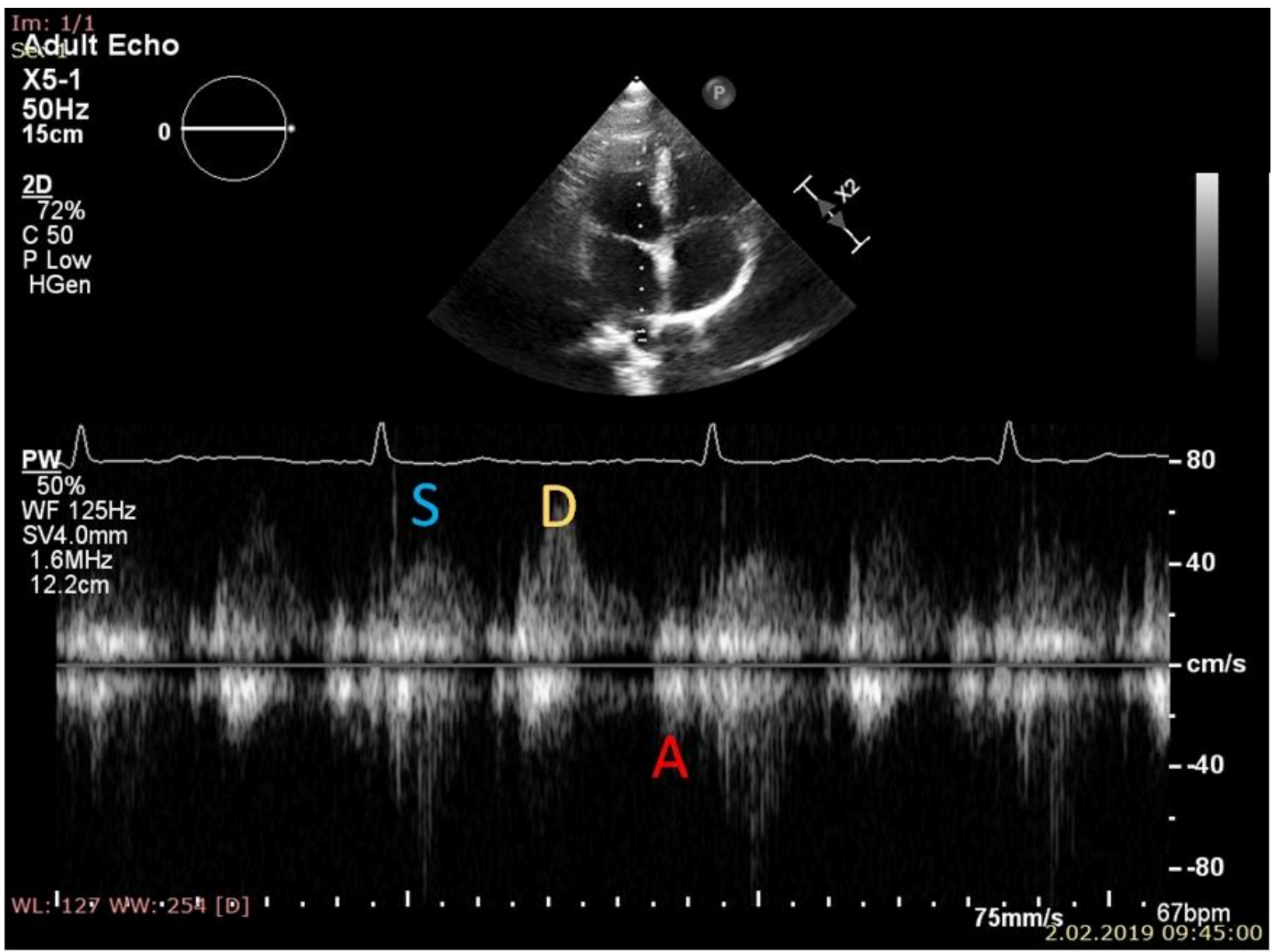

Figure 4. Normal pulmonary vein flow

Left atrial appendage has quadriphasic flow pattern: early diastolic emptying, late diastolic emptying, filling and systolic reflection waves (Fig. 5). Atrial fibrillation causes chaotic saw-tooth flow waves with varying velocities (Fig. 6). 


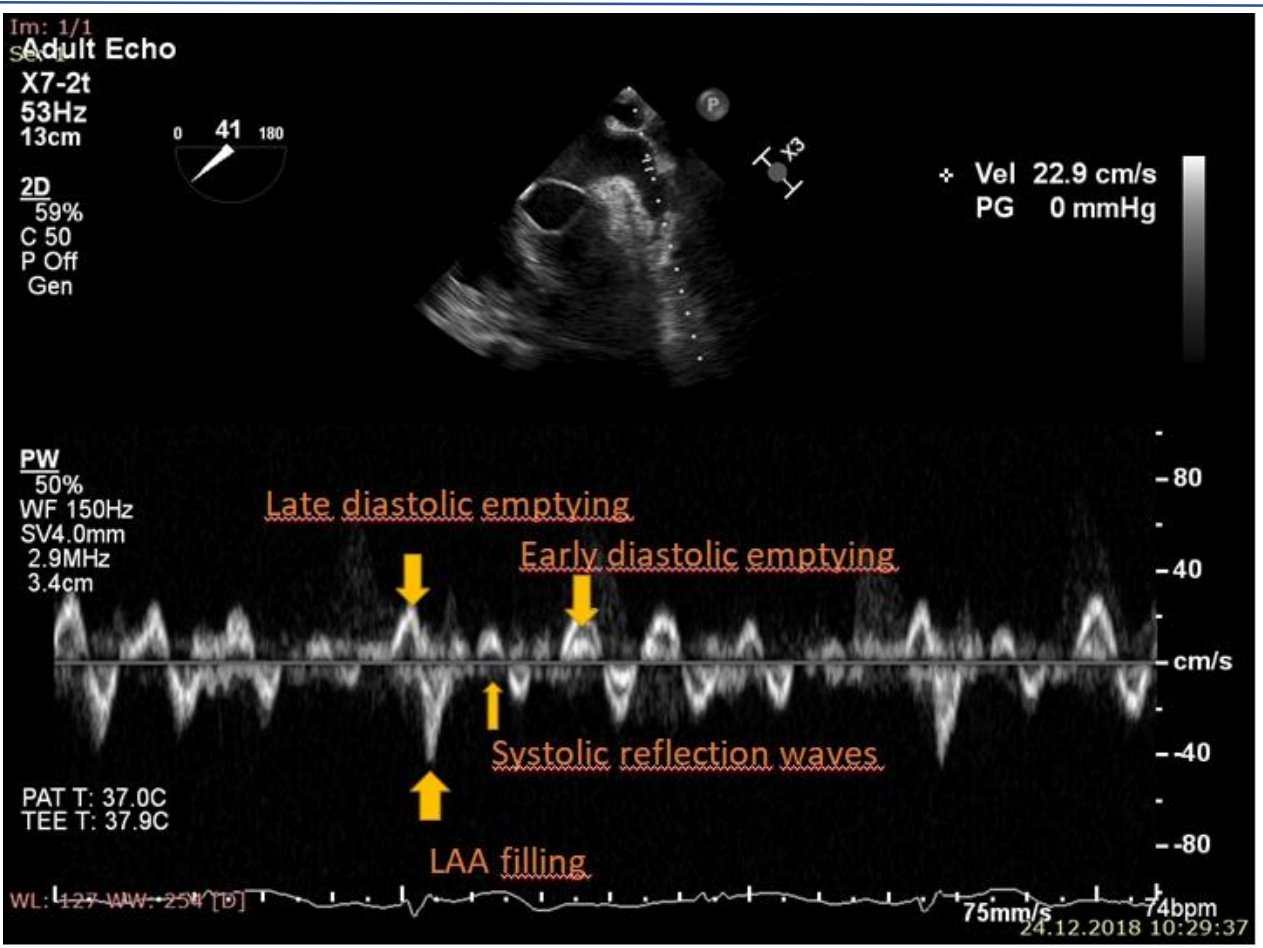

Figure 5. Normal left atrial appendage flow

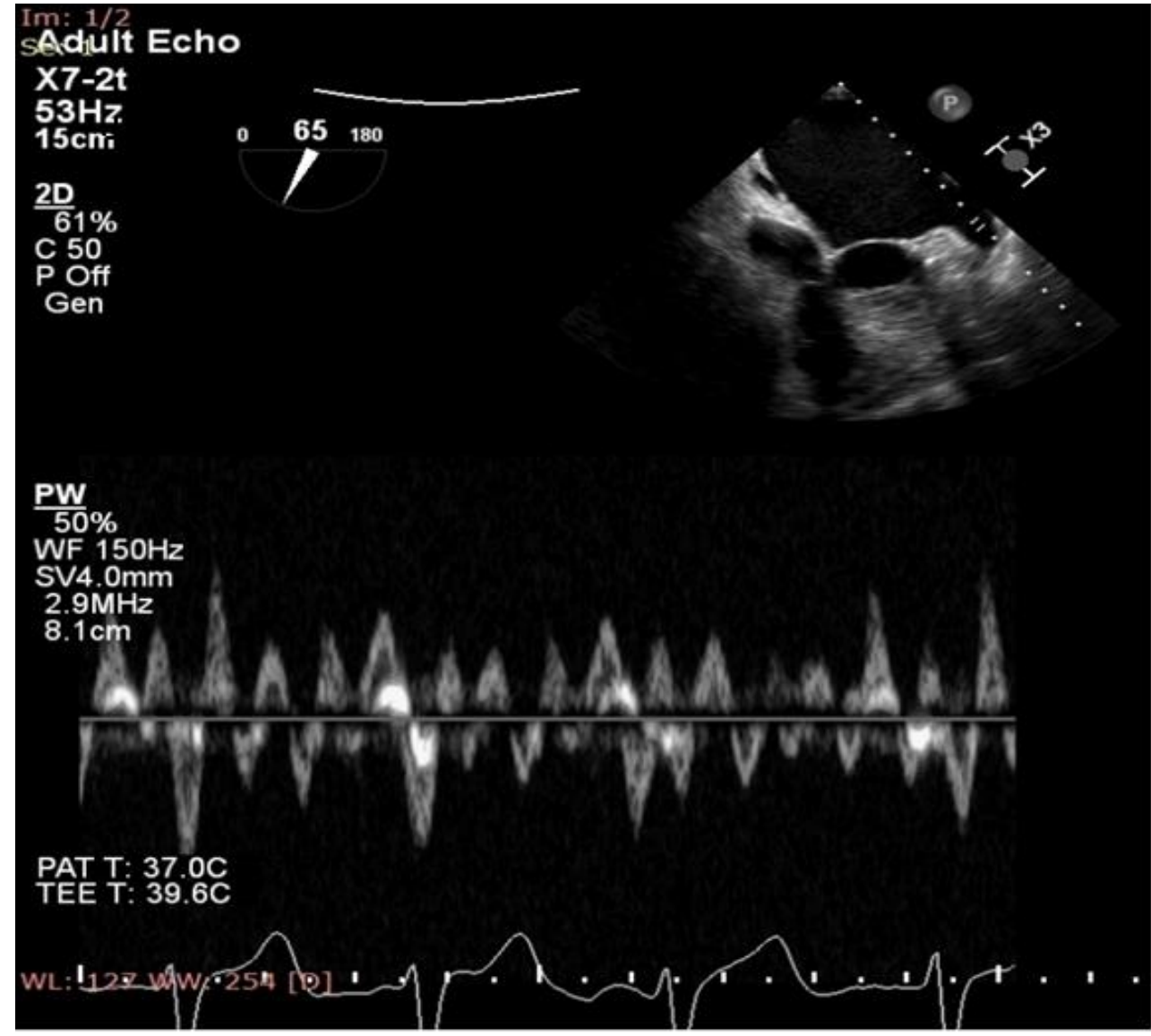

Figure 6. Left atrial appendage flow in atrial fibrillation 
Oben Baysan

Guven Hospital

Ankara, Turkey

Peer-review: Internal

Authorship: O.B.

Conflict of interest: None to declare

Acknowledgement and funding: None to declare

\section{References}

1. Fadel BM, Mohty D, Husain A, Alassas K, Echahidi N, Dahdouh Z, Di Salvo G. Spectral Doppler of the hepatic veins in rate, rhythm, and conduction disorders. Echocardiography 2016; 33: 136-40.
2. Zoghbi WA, Enriquez-Sarano M, Foster E, Grayburn $P A$, Kraft CD, Levine RA, et al. Recommendations for evaluation of the severity of native valvular regurgitation with two-dimensional and Doppler echocardiography. J Am Soc Echocardiogr 2003; 16: 777-802.

3. Yoon JY. Diastolic tricuspid and mitral regurgitation in a patient with complete atrio-ventricular block. J Clin Case Rep 2017; 07 [DOI: 10.4172/2165-7920.1000976] 